\title{
Engineering advice in policy-making: a new domain of inquiry in evidence and policy
}

Adam C.G. Cooper ${ }^{1}$, Lorenzo Marvulli², Katie Black ${ }^{3}$, John Holmes ${ }^{4}$ and Harshal Mehta ${ }^{4}$ ${ }^{1}$ UCL Department of Science, Technology, Engineering and Public Policy, $4^{\text {th }}$ Floor Shropshire House, 11-20 Capper Street, London WC1E 6JA UK

email: adam.cooper@ucl.ac.uk

${ }^{2}$ School of Social Sciences, Cardiff University, UK

${ }^{3}$ National Infrastructure Commission, UK

${ }^{4}$ UK Government Department for Business, Energy and Industrial Strategy, UK

\section{ABSTRACT}

Background. Academic research on technical advice to policy commonly focuses on social and related policy areas such as health, education and crime (Oliver et al. 2014) and disciplinary advice from science disciplines (Jasanoff 1994; Millstone and van Zwanenberg 2001). Little or no prior research in the social sciences have explored engineering expertise in policy domains where such advice is critical (e.g. energy policy).

Aims and objectives. We aim to establish 'engineering advice' as a new domain of inquiry by showing how civil servants view it as distinctive (from 'science advice'), important and similar to policy making - implying it can clash or complement it.

Methods. 18 qualitative interviews with a purposive sample of officials across a UK ministry were conducted by the authors (all but one of whom were civil servants) in 2012. The qualitative data were thematically coded to address the study aims.

Findings. A majority of officials spontaneously identified engineering expertise as both distinctive and important for their work. There was clear evidence that it both complemented and clashed with policymaking.

Discussion. We identified a range of interactions that imply a need to consider styles of management internal deployment of experts within policy organisations as well as the implications for policy making and engineering expertise given the way practices overlap. Conclusions. Further research on the ontological, epistemological nature of engineering as it relates to policy making is needed if governments and therefore society are to fully benefit from engineering advice.

Keywords: engineering advice; expertise; evidence-based policy making; science advice;

Word count: 8092

\section{Key messages}

- Engineering advice has never been properly identified and studied in the academic social science literature to date

- Engineering advice is an important and potent source of evidence in policy making in topical areas like energy policy

- In contrast to science advice, engineering advice as a practice significantly overlaps with policy practice meaning important conflicts or complementarity is possible, dependent on how the advice is deployed

\section{INTRODUCTION}


It is possible to brigade academic research on the use of technical expertise in policy-making into three major perspectives. There is a perspective based around 'evidence-

base/informed policy making' (Greenhalgh and Russell 2009; Head 2010; Nutley et al. 2007); there is a perspective around 'science advice' (Gluckman 2014; Jasanoff 2013; Pielke 2007; Schenkel 2010) and there is a perspective around what can be called 'expertise in policy' (e.g. Millstone and van Zwanenberg 2001; Page 2010). These literatures can be mapped in different ways revealing certain kinds of gaps which the study here seeks to fill. The first of these literatures is policy-topical, that is to say the policy area to which evidence or science advice or expertise is being fed into. The second is (academic) disciplinary source of the expert knowledge or evidence. We explore these areas briefly below with a view to making the claim that engineering advice has not been studied as a phenomenon within these domains.

\section{Policy topics}

'Evidence-based policy making' is most closely identified by social and health policy areas, including education and criminal justice domains. This is evident from, for example, mapping the categories of returned articles from a Scopus searchi for the phrase "evidencebased policy making". This returned 488 articles from 1997 (prior to which no instances are returned), where the top subject area is 'Social Sciences', followed by 'Medicine'. Looking at the top 10 most cited papers, 4 of the paper's titles were concerned explicitly with health or social policy domains such as education or early years provision. The most highly-cited paper in this domain, by lan Sanderson, references explicitly "crime prevention, employment and welfare policy, health, education and local government" (Sanderson 2002: 9). Finally, in Oliver and colleagues' influential systematic review on the effective use of evidence by policymakers (Oliver et al. 2014) the predominant domain was health, with other sectors, including transport, far behind.

'Science advice' and 'expertise in policy' also often tend to focus on health-related topics (including epidemics, xenotransplantation (Weale 2001) or antimicrobial resistance (Spruijt et al. 2014)) but also are concerned with other physical science-oriented areas such as agricultural biosciences (e.g. GM crops, and diseases in cattle: Millstone and van Zwanenberg 2001; Oreszczyn 2005; Schwach et al. 2007). A similar Scopus search as per above for the phrase "science advice" returns 141 articles stretching back to 1963 with a similar topical orientation in the titles of the top 10 most-cited papers.

In summary, policy areas which reflect engineering expert interest are very much in the minority in the literature in Scopus, in favour of topics which foreground scientific expertise.

\section{Disciplinary sources of evidence and expertise for policy making}

Given the topical expertise and the nature of the context in which that expertise and evidence is being offered, it is simple to infer that a significant orientation of these studies is around either social science (as in social research expertise, (Cooper 2016; Kattirtzi 2016) or around natural, medical or physical sciences. Certainly, in Page's study of expertise and policy bureaucracies, he emphasizes economics, veterinary science, epidemiology, marine 
biology, materials science, medicine and chemistry: all in line with the policy topical areas identified above (Page 2010).

The upshot is that in almost no instances are the engineering disciplines mentioned in these studies. Searches in Scopus for 'engineer*' within the phrasal search "evidence-based policy making" returns 49 hits, but closer inspection of abstracts reveals that none of these is focused on the role of engineers in delivering 'evidence-based policy'. Similarly, phrasal searches for "engineering advice" returns 33 hits but closer analysis of the incidence of 'engineering advice' in the abstracts reveals the use of the phrase to be descriptive of an aspect of the projects reported (e.g. "Some engineering advice is proposed based on the numerical calculation with the different random excitation inputs" from Li et al. 2009) rather than being an object of study. This impression is further reinforced by appraisal of the subject areas Scopus has classified the output, with 25 of the returns identified as from Engineering and with social sciences not identified at all.

The only instances we found of 'engineering advice' being considered as a practice in the context of evidence-based policy making or science advice are in a chapter of (Doubleday and Wilsdon 2013) Future Directions of Science Advice in Whitehall where Brian Collins reflections on engineering policy making and advice (Collins 2013) and in a policy document from the UK Engineering Councilii. We return to Collins' analysis below when we explore the difference between science advice and engineering advice. Either way, it seems clear that 'engineering advice' has not been the target of academic social science inquiry to date. This may be due to engineering and science being brigaded under a common title of 'science', such that all 'science advice' literature is implicitly understood to include 'engineering advice'. However, this assumption relies on there being no important differences between engineering and science in relation to policy making. We explore this question below.

\section{Engineering and Science: is there an important difference?}

An underpinning assumption made in this study is that 'technical advice to policy' (the phrase used in the opening line of this paper) can comprise distinct disciplinary perspectives on the part of the expert or the evidence being used. This is likely uncontentious (though evidence itself can sometimes be drawn from inter-, multi- and transdisciplinary studies). A further assumption is that there is merit in distinguishing between the different disciplinary perspectives. The major distinction is between the natural or physical sciences and the social sciences. This distinction commonly plays out as a kind of epistemic battleground or site of complex negotiation for evidence-based policy making (Cooper 2017b; Cooper 2017a; Lowe et al. 2013; Maxwell 2014; Shove 1998; Sovacool et al. 2015). This is as evident in the domain of energy policy as it is in climate and environment. A disciplinary distinction that is apparently seldom in the evidence-based policy literature is between science (physical or social) disciplines and engineering disciplines. Here, we draw that distinction and argue why it matters when considering technical advice to policy.

The Royal Academy of Engineering, the UK's umbrella institution for engineers of all disciplines, answers the question 'What is Engineering?' by saying "Engineers make things, they make things work and they make things work betteriii". This contrasts with common definitions of science which includes elements like "the systematic study of the ... world 
through observation and experiment" (our emphasis). While there is significant overlap in the nature of the two disciplinary domains, the goal of scientists is to study the world with a view to understand it, while the goal of the engineer is to make something so that some aspect of the world is better. The both rely on systematic study of the world and will deploy forms of observation and experiment in their practice, but the goals of each are quite different. Science's perspective is about discovery and to that extent is why those writing about science advice will often focus on notions of 'balance' (Gluckman 2014), 'unbiased' (Schenkel 2010) and being an 'honest broker' (Pielke 2007). Clearly, the distinct epistemic and ontological perspectives of engineering, science and policy are crucial here, but limited space prevents deeper analysis here.

The emphasis engineering places on 'making' and 'better' reflects normative concerns that align with policy practice: policy officials are also 'making' and aim for 'better'. The UK Government's website for 'the Analysis Function of Government' (the part most relevant to using scientific and engineering expertise) asserts that:

Integration of analytical techniques and scientific methods into the business of government helps achieve better outcomes for decision makers and the public. That is why analysts are needed and based in every government department. ${ }^{\text {iv }}$

Further, their intention in doing that 'making' reveals a shared normative stance with engineering, policy officials also aim to 'improve' aspects of life: standards of living, quality of life or wellbeing, or economic growth and related indicators $v$. This close alliance between the aims and nature of engineering and policy making indicates the two can be either highly complementary, or competing and confusing if operating in the same organisation. It raises questions of whose 'better' is pursued, and which expertise is prioritized in policy making? This focus on making aligns with Schmidt's use of willing versus knowing: science is about knowing the world whereas engineering is "about how the world will be in the future." (Schmidt 2014: 105). This of course also echoes the nature of policy making.

In Collins' paper identified above, his reflections on engineering advice for policy (and the demise of it in the UK over the last 50 or so years) identify concerns with how technical advice is being under-used in these settings and there being a loss of what he calls 'policy execution expertise' (p. 73). He notes that "successful execution demands engineering expertise" - echoing the tension identified above regarding the potential complementarity of engineering expertise and policy expertise. The broader question that arises from Collins' analysis is how to do engineering advice effectively. And within that, how to manage engineering expertise with policy expertise.

By contrast, we want to take a step back from Collin's argument and raise the notion of 'engineering advice' per se as a particular embodied practice that is sufficiently distinct from 'science advice' (Gluckman 2014; Jasanoff 1994; Pielke 2007; Weale 2001) and other approaches outlined above to warrant special scrutiny. Here, then, the hypotheses being explored are:

a. That engineering advice is distinct and can be distinguished from science advice

b. That engineering advice has features that make is particularly important for policy and therefore demands critical scrutiny of it as a practice 
c. That the overlap between engineering and policy practice could lead to either disruptive competition or effective co-operation between the two groups.

Here we explore this issue through a small study of policy and engineering officials based in the UK's former energy ministry, the Department of Energy and Climate Change (DECC).

\section{Understanding the effectiveness of engineering advice in DECC}

\section{Background}

The research was initiated by Cooper, during his time as the Head of Social Science Engagement at DECC (2011-2013). He was part of the 'Evidence Team' which was set up by the Chief Scientist with a view to improving the use of evidence within DECC. This team was placed, organizationally in a part of DECC called the Science and Innovation Group (SIG), which itself was part of the Strategy and Evidence Group (SEG). Shortly before their arrival a new engineering team in SIG had also been recruited and it was in this context that an internal study was approved to look at how the new engineering team was working with the policy officials they were employed to advise. The data collection all took place in the summer of 2012.

\section{Methods}

The general approach was to capture narratives and perspectives from DECC policy officials regarding their working experiences with internal engineer officials. The focus therefore was on how well formal, internal engineering advice was received by those tasked with putting together policy proposals for ministers to approve. We therefore used a standard semistructured interview format which enabled us to collected reasonably consistent answers against core issues but provided enough openness to allow issues narratives to emerge naturally.

\section{Sampling}

We purposively sampled mid-level policy officials - those at the civil service grades between Higher Executive Officer and Grade 6 where most of the policy work is done (Marvulli 2017; Page 2003; Stanley 2000; Wilkinson 2011). We chose officials working across a range of different policy teams. This covered the two main policy directorates at the time (Energy Markets and Infrastructure and International Climate Change and Energy Efficiency) and 7 of different policy programmes out of a possible 11. This ensured good coverage across the department in the areas where the most interaction ought to have been. The logic here was that if we found no or poor collaboration in these areas it was unlikely to be any better elsewhere.

Table 1 sets out the achieved sample, which comprises 18 officials in total covering grades from HEO to Grade 6 across different policy areas ${ }^{\text {vi }}$. The majority are Grade 7 - the mainstay of policy making in UK government and 8 have had long experience in the civil service. One of the Grade $7 \mathrm{~s}$ with an engineering background was an embedded engineering official 
(Code [6AC]) who sat day-to-day with the policy team but who had previously been part of the engineering group in SIG.

[Table 1 about here]

Interview schedule

Marvulli and Cooper designed the research instruments for data collection (topic guide) and Marvulli led a brief interview training session for the team, prior to data collection. Interviewers were instructed to make use of elements of the Active Interview style of questioning (Holstein and Gubrium 1995), with its stress on the collaborative nature of meaning production in the interviewer/interviewee interaction and on the incitement to produce a 'narrative' account of one's own working life (see e.g. Whitaker and Atkinson 2019). The method taps into the respondents' stock of competent experience of work, with a view of discussing details of the activities individuals needed to do to get work done. It was adapted to capitalise on the existing rapport between participants and most of the interviewers, and the consequent sharing of understandings around working practices and jargon. Structured in such way, the interview method worked as a magnified version of the 'interview to the double' strategy (Nicolini 2009).

Data collection

Participants were recruited, and interviews arranged, by email by the interview team member who was assigned to undertake it. Commonly, interviews would take place in meeting rooms at DECC or sometimes spare spaces such as in a corner of the café. Two interviews, conducted by Marvulli were done by phone. All interviews were conducted in line with the UK Data Protection Act.

\section{Data analysis}

All data analysis was handled by the lead author. The transcripts for all participants were first separated into two groups: those respondents with an engineering role or background $(n=3)$ from those with a policy role or background $(n=15)$. Then transcripts were reviewed and particular quotes that related directly to engineering advice were identified, and a simple thematic analysis was then undertaken.

\section{Results}

Here we report only on the challenges and benefits identified by all participants and provide a brief analysis of the range and sub-types identified with each. Overall, every participant noted both benefits and challenges of working across the expert-policy relationship. These statements were then further reviewed to look for examples that seem to be particular to engineering advice as a phenomenon and how that might manifest different benefits and challenges from wider evidence-based policy making issues identified elsewhere in the literature. Individual participants are identified by a code based on who interviewed them, and a short description of their background and role. 
Around three-quarters of the participants made statements that recognized at least the distinctiveness of engineering advice or the importance of it. We assumed that if the participant made a statement mainly about importance, it was also coded as recognizing the distinctive nature of engineering advice. These statements were made by participants from a range of different grades and with a mixture of experience in the civil service. Specific parts of the quotes which highlight the notion of distinctiveness, either in terms of the nature of engineering advice (in particular the use of words like 'technical' or, ironically, science-related terms), the difference it makes to the day-to-day work in relation to the effective deployment of engineering skills or knowledge (e.g. engineering terms like 'temperature', 'design' or technologies) are emboldened.

Having said that, the piece of work that I described earlier where we're trying to establish just what the risk and probability of leakage is for policy reasons, to do with encouraging people to invest in [carbon-capture and storage], so to get this debate on a rational, technical level, l've got no idea whatsoever whether the work that has been provided to me, and ultimately we are going to publish, stands up technical scrutiny.

[13JH] Grade 6 policy, long experience

In my current role then I'm expected to be the policy expert but I'm not expected to be a technical expert on combined heat and power so I do need to make sure that colleagues in the science and evidence group are content with the evidence we're drawing on that it is robust from a technical perspective and that we are making decisions based on correct information and not on selectively quoted information or a buyer's perspective

[22LM] Grade 7 policy, long experience

Or if you wanted to link to existing schemes, that one could be running at a different temperature to that one, they could be designed in different ways for different purposes.

[8AC] Grade 7 policy, medium experience

The quotes above show how engineering advice is distinctive, dealing specifically with objects that could "designed in different ways" or "be a technical expert on combined heat and power" and questions of technical performance, as in the first case of carbon-capture and storage policy needing to estimate engineering performance of storage options. Science advice, as understood in the literature on science advice would have very little to say about the (technical) performance of different technologies, whereas policy officials here are directly turning to sources of engineering advice for exactly those kinds of questions. In many respects they are, indirectly drawing on the science of efficiency analysis and underpinning physics that it might rely on, but there is a sense here of the officials being concerned with designed and built objects as opposed to what the evidence is about the why or how certain phenomena might function. As one Grade 7 official put it when talking about the utility and distinctiveness of the engineering experts: 
it's very constructive and it means they can bring something to the table that we wouldn't otherwise have because they've got an understanding of the technical standards that need to be delivered and the way in which we might do those;

[16HM] Grade 7 policy, medium experience

This participant here is clear that the presence of engineering officials is expressly distinctive - something they "wouldn't otherwise have" - and that the distinctive nature of that advice is linked causally to the "technical standards" and "the way" (i.e. design) such technical standards might be deployed. This was reinforced by another official, one with an engineering background, contracted in specifically to provide internal support to policy development:

Yeah, so I think the role of the technical work stream is effectively to define requirements that are deliverable. So we don't want to define a solution, but at the same time we don't want to specify requirements ... which cannot be delivered.

[25LM] Contractor Grade 6/7 policy, short experience

The participants that didn't mention engineering advice directly tended to discuss other issues relating to challenges in working with different analysts (including economists) or the issue of the geographic separation of the SIG team from other policy tea ms (in the main DECC offices in London, SIG occupied a corner of the 6th floor alongside other analysts from SEG, while policy teams were arranged across lower floors). One policy official, at the time of being interviewed had not actually heard about the engineering team, despite the body having been in place for around a year, foregrounding a basic issue found elsewhere in the wider evidence-based policy making for effective use of expert advice: make it visible and accessible (Oliver et al. 2014).

Never heard of [engineering team leader], didn't know there was an engineering team

[1KB], Grade 7 policy, medium experience

I don't think that demand-side response involves lots of technical expertise. Say, storage is a lot more technical in how it works and stuff like this.

[23LM], Grade 7 policy, long experience

The second of these respondents was also unclear about the role of SEG, but also felt that their policy area didn't require a lot of technical expertise, so did not refer specifically to engineering advice as important, even though they recognized it as a distinct resource, required elsewhere. This official had no engineering background, so it is possible that their interpretation of their policy domain and its reliance or otherwise on engineering advice is in some way a function of that epistemic perspective.

Importance of engineering advice

A smaller number - six interviewees - made statements that were easily understood as identifying the importance of engineering advice in particular to undertaking their policy work effectively. This included recognizing that they needed to discuss particular issues with 
those that can provide such expertise and that there tended to be too few overall. This is not a distinctive feature of engineering advice per se, but reveals a recognition that when provided it is not only useful as is, but that there is significant demand for it, indicating the general importance of this style of advice in particular areas of policy making.

... saying they've got an available resource and then being taken on to something else by their Grade 6 at the last minute left us a bit in the shit with sorting out the assessment side of things.

[11JH], HEO policy, long experience

Here this HEO shows how directly the engineering advice is to the actual formation of policy, since without that resource, their team are unable to complete their work satisfactorily. The emphatic use of the phrasing indicates how fundamental this resource is seen.

\begin{abstract}
And so the technical support that we need is basically understanding the technologies because I'm not a technologist, I'm a policy person, so you can work with the industry but you need to be discerning if some of what they're saying is right or wrong. If you're looking at, for instance, standards: have we gone far enough? Is that really the right thing to be doing at this particular time? Is that going to help to improve the performance of a heat pump and that sort of thing? So the technical aspects are important from that point of view.
\end{abstract}

[7AC], Grade 7 policy, long experience

The quote from the Grade 7 above demonstrates the importance of engineering advice, focusing explicitly on technical, non-scientific expertise that goes beyond policy knowledge into engineering. This includes a focus on standards to help support the performance of heat pumps, as well as interpreting the position of industry actors. This point links through to the importance of engineering advice in policy evaluation in this context, a point reinforced by testimony from the embedded engineering official [6AC] examined more closely below. They noted:

...the very small policy I work on is [an energy policy], and it's designed in a way now where the evaluation side - so us going out and actually measuring how well stuff is performing - is an absolutely crucial part of the policy

I think historically [technical evaluation] would've just been tacked on because someone on the sixth floor said "You haven't done this" or "You need to do this". And the first phase that's exactly how it was done, it didn't work very well. And the second phase I think I'm relatively confident because of my influence it is totally integrated, and even while I was doing it my policy colleagues were saying "This is very complicated, this is difficult". "I know it's difficult but we've got to do it otherwise it's not going to work". And it's only because I was embedded I think that I managed to do that.

[6AC], Grade 7 engineering official, medium experience 
These quotes illustrate the foundational role engineering advice played in establishing a policy that "works". The key barrier to that was (in the view of [6AC] - the embedded engineer) was the application of technical knowledge (characterized as "complicated" or "difficult" in reporting the views of non-engineering officials), again hinting at the notion of reliance on a set of knowledge and skills embodied by engineering expertise that was provided by the engineering official.

On another occasion, a respondent highlighted the valuable role of external engineering advice (that is, engineering advice provided via those outside the civil service).

Interviewer: And what about externally, you said they were quite good at getting in external experts, so did your team commission external work and can you talk to me a bit about how that went if you did?

Respondent: Well, we didn't commission external work but we set up an external advisory group, so we basically got to a position where we were unsure of how to deal with uncertainty in how much insulation could save, so it was recommended, possibly, I'm not actually sure where the recommendation came from, it might have been from the scientists, but anyway, to set up this panel who with engineers and academics and engineering companies to advise us on the level of uncertainty and what might be feeding that uncertainty. So, the policy team was in charge of setting that up and running it and the scientists sat in on it as well.

Interviewer: Okay, and was that positive, did that help?

Respondent: Yes, it helped because was a controversial area, basically saying that solid wall insulation doesn't deliver what we expected it to do, which is a difficult message, and so it was vital to have a bit more external credibility, so yes, it was.

[2KB], Grade 7, policy, short experience

Again, this testimony from a policy official recognizes the important role of engineering advice per se. As is often seen with science advice, external advisory panels are sometimes required to enable more effective advice in areas considered "controversial". This point is recognized by the official here, indicating that for all the differences identified between science and engineering advice, both gain credibility - and with it, influence to change policy - when sourced from extra-organisational (typically academic) sources (Cash et al. 2003). It is also worth reflecting on the level of investment required by the policy official to set up such a panel. The time and effort to do so further illustrates the degree of importance placed upon this source of expertise in this context.

Engineering advice as complementary or competing with policy making?

A fundamental argument is made here regarding the distinction between science advice as generally understood in the literature and the practice of engineering advice in policy. Here participants in the study, including and in particular the embedded engineer [6AC] referred to by other participants, give insights into how engineering advice overlaps with policy making practice. Commonly policy officials identified areas where engineering advice 
seemed to compete with policy making as if on a separate but similar track. For instance, one policy official recalls discovering some work being undertaken by the engineering analysis team in SIG, and framing it as them 'solving their [policy's] problem':

but it's almost like... "Oh," you know, "they're almost solving our problem for us and we didn't even know that piece of work was going on."

[18HM], HEO-D policy, short experience

This illustrates a few inter-related issues: the distance between teams (physically and institutionally) leading to lines of policy development occurring in parallel rather than tied together, but also the notion that engineering teams can carry out work that solves a policy problem, as opposed to just providing evidence that might suggest one or other solution. Similarly, another official paints a related picture:

Again, you see, our economist has said, "what analysis do you want me to do there?" But now we're speaking to SIG, and they're like, "oh, well, in this competition, we didn't do any of this, but we'd be using such-and-such to do $X, Y$ and $Z$." So, it's almost like now our own economist has been chopped out of the process, and although it's been central... I don't know. I just feel there should be more joining up, perhaps.

[11JH], HEO policy, long experience

No, it's seen as a potential for total overlap. I mean it worked really well with [one engineering official] but then we got told that an [other] engineer was starting ... And he thought he was doing our job, so he came down and said 'I'm looking at this, this and this'. And we were like 'er, that's what we're doing' and the same things with other people in the Strategy team as well. So, I think, with [one arrangement] it worked really, really well: it was an additional resource making the evidence base and the work, much more sound. But there was a strong potential for people to just go off and duplicate and do their own thing because they don't like what policy teams area doing. And that's been more my experience than the [positive experience] and that's where I think it falls down because it becomes very inefficient for the department.

[19AC], Grade 7 policy, medium experience

So again, parallel engineering official-led, but closely-allied policy processes have been executed which end up being competing with the policy-owned process. Again, part of this is perhaps due to structural approaches to organizing engineering officials - since respondents here are referring mainly to the centralized team placed on the $6^{\text {th }}$ floor of the ministry's main building, separate to policy teams. The second testimony above refers to a positive, complementary approach where the engineering advisor was effectively placed in her team:

Respondent: ... I asked [the engineering official assigned to support the official] to dig to find that evidence base. I set the criteria for the evidence base if that's what you mean. But I mean we did it in collusion - I wasn't sitting there going 'and go away and do this' - 'this is the table, what you do think?' 
Interviewer: So, it was a collaborative effort?

Respondent: So, collaborative in terms of getting the contacts and working out what should happen next. And also, if [the engineering official assigned to the work] got stuck or didn't know how to answer a question we'd sit down and try to work out a way forward for her. So, I suppose I was her manager in that sense. Rather than it being a partnership.

[19AC], Grade 7 policy, medium experience

This is similar to effects reported by (Wilkinson 2011) for scientists in sister UK department Defra, indicating another point of similarity between science and engineering advice - both are affected by organisational form (Boutellier et al. 2008; Irving et al. 2019; Kabo 2016).

From the perspective of the embedded engineer [6AC], examples of both competing and complementary processes are evident from their testimony. This embedded official starts by identifying two key elements: that their move to being embedded was helpful (as evidenced by spontaneous testimony above) and that such a move was consistent with them being involved directly in core policy making - indicating a complementarity of engineering advice with policy practice.

...I sat with the policy team and mainly because I thought that was how I could be most effective with them, and I think they appreciated that.

So policy making seems to be made, quite a bit of it at least, across the desk not necessarily in writing; so the real 'idea bouncing around' stage often starts verbally across desks and if you're not there physically to hear that then I think you're then waiting for someone to write something and it to go around all the policy colleagues and then be sent around the department.

[6AC], Grade 7 engineering official, medium experience

There are few more strategic places to be involved in policy making than in the 'idea bouncing around' stage where the base options of what to do are identified. In contrast to science advice and the advice given to science advisors by experience Chief Scientists such as Sir Peter Gluckman - where he suggests scientists should 'expect to inform policy, not make it' (2014), here the engineer is very much in the 'making it' phase.

A key element of the way in which engineering advice informs energy policy is demonstrated by the following testimony from this embedded engineer [6AC]:

So it's been a relatively positive experience and I'm kind of finding that the policies that are being made now are I think at least - the very small policy I work on is [an energy policy], and it's designed in a way now where the evaluation side - so us going out and actually measuring how well stuff is performing is an absolutely crucial part of the policy

[6AC], Grade 7 engineering official, medium experience 
Here in a sense is an example of how engineering policy has led directly to certain aspects of the policy being made in certain ways: there are objects in the policy that would not otherwise exist but for the engineer's advice. Interestingly too, this advice fits into what Cooper has called 'process advice' - here 'engineering process advice' as opposed to science process advice (Cooper 2016). Revisiting a comment by the embedded engineer [6AC] we examined earlier, we see this individual drawing a contrast with the past and providing 'engineering process advice' that leads to an integrated technical evaluation of the policy:

I think historically [technical evaluation] would've just been tacked on because someone on the sixth floor said "You haven't done this" or "You need to do this". And the first phase that's exactly how it was done, it didn't work very well. And the second phase I think I'm relatively confident because of my influence it is totally integrated, and even while I was doing it my policy colleagues were saying "This is very complicated, this is difficult". "I know it's difficult, but we've got to do it otherwise it's not going to work". And it's only because I was embedded, I think that I managed to do that.

[6AC], Grade 7 engineering official, medium experience

The results presented in this section illustrates how - in this particular context - engineering advice is understood and acts in a way distinctive from science advice, how that advice is deemed important to policy officials, and how it - as a practice - significantly overlaps with policy practice. In contrast, perhaps to science advice, engineering advice - as recognized by the officials who experience it, can both compete with and complement policy-making by policy officials. It is clear that certain organizational forms are important in how these tensions are managed. We now go on to discuss the implications of this.

\section{Discussion}

The argument made in the earlier sections of this paper is that engineering as a discipline (or group of disciplines) is sufficiently distinct from science such that in the practice of expertise and evidence informing policy, it deserves distinct treatment from scholars in this area. This claim was supported by the observation that mainstream evidence-based policy making literature makes little substantive reference to engineering advice (and with it rarely, if ever explores policy domains where such advice is central, such as energy policy). Similarly, the term 'engineering advice' has not been deployed in the same way as 'science advice' (that is, to capture a specific set of practices in policy making) and therefore does not currently exist as an object of inquiry in the same way.

The core hypotheses underpinning the claims in this paper were then explored in relation to a dataset collected by the authors undertaking an internal study within the UK energy ministry, DECC, in 2012. We identified a set of testimony that illustrates how practicing officials see the distinctiveness, importance and overlapping nature of engineering advice with policy practice. While only illustrative at this stage - and clearly further, more structured inquiry is required to unpack and examine the claims here in more detail in different settings - what emerges is a picture of engineering advice as a practice that has both commonalities with science advice but also clearly distinct features. 
The commonalities between science advice and engineering advice are that both are often performed via similar practices and suffer similar challenges according to organizational form. Examples above reflect the three tableaux identified by Page (2010), showing bureaucrats as experts, mobilisers of experts and, to an extent, servants of experts. Further, in line with Wilkinson's experience, the institutional forms can interfere with the practice of engineering advice, with the result of creating divisions between the scientific or engineering experts and those directly responsible for policy making (2011). The upshot of these arrangements reported here are either a lack of use of expert advice or a problematic parallel-working arrangement, leading to 'inefficiencies', or highly-effective practice whereby expert engineering advice is taken on board at the policy making stage.

This latter point raises a key point of distinction between engineering advice and other forms of knowledge utilization: the common discourse and focus of the latter is on how to improve the use of evidence in policy. A recent systematic review by Oliver and Cairney identified 86 papers focused only on the research advising academic experts (including 'science' and 'scientists' in particular - but not engineers) on how best to influence policy (Oliver and Cairney 2019). The clear implication is that bringing expert knowledge to bear on policy making is challenging even when one takes into account the role of politics and values in policy making (Parkhurst 2017). What is important here is the availability of clear examples of the effective use of expertise in policy-making. The clearest is the impact of the embedded engineer [6AC] shaping the evaluative capacity of a policy as a direct consequence of their entrepreneurialism (Mintrom and Norman 2009). This supports some of the advice as effective by Oliver and Cairney, but also suggests that such practices may be relatively common within UK ministries. At the same time, there may also be something particular about engineering as a discipline that makes this effectiveness more likely. The ability to answer questions about real-world effects, and - speculatively - the relationship engineering as an academic discipline has with engineering business (note that one of the three engineering-trained officials was a consultant contractor, which was not unusual in the author's experience at DECC) puts these experts in a different, perhaps more pragmatic class of expert, making their knowledge more in demand, and potent, for officials aiming to make policy that works.

If engineering expertise is more 'potent' a form of expertise for policy-making due its inherent problem-solving, real (physical) world orientation, this may explain in part its problematic dominance in policy-making for energy (e.g. Sovacool 2014). One next step then is to explore how best to deliver a form of engineering expertise that leverages any inherent influence while mitigating these negative consequences. One option here is for a more integrated, interdisciplinary form of expertise, reliant on socio-technical research knowledge base (Cooper 2017b; Cooper 2017a), underpinning a new, transdisciplinary approach in engineering advice (Lang et al. 2012).

\section{Conclusions}

We propose to introduce a new term into the lexicon of evidence-based policy making research: "engineering advice". This, we argue, is related to, but importantly distinct from "science advice" and other related objects of inquiry in the literature. We illustrated the case for this with reference to interview data from a study conducted by the authors, most 
of whom were employed as civil servants at the time, with policy and engineering officials in DECC in 2012. From this data, the distinctiveness, importance and challenges and benefits of employing engineering advice was observed, described spontaneously by officials working within the engineering-heavy energy policy setting. Further work is proposed on how to better understand engineering advice in practice, and identify what guidance might be created to support good practice in this area.

Research ethics: Research ethics was formally obtained via Marvulli and the Cardiff University Research Ethics Committee in May 2012. Internal consent from the then DECC Chief Social Researcher to conduct the interviews in line with Government Social Research guidelines was obtained by Cooper in April 2012.

Contributor statement: $A C$ and LM wrote the first and subsequent drafts of the manuscript, with comments from JH, KB and HM. AC conceptualised the study. AC and LM designed the study. All authors took part in interviewing equally. $A C$ and $L M$ conducted data analysis and interpretation, with contributions from $\mathrm{KB}, \mathrm{JH}$, and $\mathrm{HM}$.

Acknowledgements: The authors would like to thank the reviewers for their insightful and helpful comments in helping make this paper significantly better. Also to Prof Paul Cairney for his thoughts on an earlier draft and comments from the audience and other presenters at the IPPA conference, Montreal 2019.

\section{REFERENCES}

Boutellier, R., Ullman, F., Schreiber, J. and Naef, R. (2008) 'Impact of office layout on communication in a science-driven business', $R \& D$ Management 38(4): 372-391.

Cash, D. W. et al. (2003) 'Knowledge systems for sustainable development', Proceedings of the National Academy of Sciences 100(14): 8086-8091.

Collins, B. (2013) 'Engineering policy: evidence, advice and execution', in R. Doubleday and J. Wilsdon (eds). Future Directions for Scientific Advice in Whitehall. pp. 73-78, available at http://sro.sussex.ac.uk/47848/.

Cooper, A. C. (2016) 'Exploring the scope of science advice: social sciences in the UK government', Palgrave Communications 2(16044): 1-9.

Cooper, A. C. G. (2017a) 'Building a socio-technical energy research community: Theory, practice and impact', Energy Research \& Social Science 26: 115-120.

Cooper, A. C. G. (2017b) 'Building physics into the social: Enhancing the policy impact of energy studies and energy social science research', Energy Research \& Social Science 26: 80-86.

Doubleday, R. and Wilsdon, J. (2013) Future directions for scientific advice in Whitehall, available at http://sro.sussex.ac.uk/47848/ (accessed August 2015). 
Gluckman, P. (2014) 'Policy: The art of science advice to government', Nature 507(7491): 163-165.

Greenhalgh, T. and Russell, J. (2009) 'Evidence-Based Policymaking: A Critique', Perspectives in Biology and Medicine 52(2): 304-318.

Head, B. W. (2010) 'Reconsidering evidence-based policy: Key issues and challenges', Policy and Society 29(2): 77-94.

Holstein, J. and Gubrium, J. (1995) The Active Interview, 2455 Teller Road, Thousand Oaks California 91320 United States of America: SAGE Publications, Inc., doi:10.4135/9781412986120.

Irving, G. L., Ayoko, O. B. and Ashkanasy, N. M. (2019) 'Collaboration, Physical Proximity and Serendipitous Encounters: Avoiding collaboration in a collaborative building', Organization Studies 0170840619856913.

Jasanoff, S. (1994) The Fifth Branch: Science Advisers as Policymakers, New Ed edition., Cambridge, Mass.: Harvard University Press.

Jasanoff, S. (2013) 'The science of science advice', in R. Doubleday and J. Wilsdon (eds). Future Directions for Scientific Advice in Whitehall. pp. 62-68, available at http://sro.sussex.ac.uk/47848/.

Kabo, F. W. (2016) 'A Model of Potential Encounters in the Workplace: The Relationships of Homophily, Spatial Distance, Organizational Structure, and Perceived Networks':, Environment and Behavior, doi:10.1177/0013916516658501.

Kattirtzi, M. (2016) 'Providing a "challenge function": Government social researchers in the UK's Department of Energy and Climate Change (2010-2015)', Palgrave Communications 2: 16064.

Lang, D. J. et al. (2012) 'Transdisciplinary research in sustainability science: practice, principles, and challenges', Sustainability Science 7(1): 25-43.

Li, W.-M., Zhu, H.-P., Tu, J. and Xia, Y. (2009) 'Operational bridge responses due to road surface profile spectral excitations', Tumu Jianzhu yu Huanjing Gongcheng/Journal of Civil, Architectural and Environmental Engineering 31(1): 72-76+88.

Lowe, P., Phillipson, J. and Wilkinson, K. (2013) 'Why social scientists should engage with natural scientists', Contemporary Social Science 8(3): 207-222.

Marvulli, L. (2017) Towards sustainable consumption: an ethnographic study of knowledge work and organisational action in public policy development and implementation. phd. Cardiff University., available at http://orca.cf.ac.uk/105518/ (accessed September 2019).

Maxwell, K. B. (2014) 'Getting there from here', Nature Climate Change 4(11): 936-937. 
Millstone, E. and van Zwanenberg, P. van (2001) 'Politics of expert advice: Lessons from the early history of the BSE saga', Science and Public Policy 28(2): 99-112.

Mintrom, M. and Norman, P. (2009) 'Policy Entrepreneurship and Policy Change', Policy Studies Journal 37(4): 649-667.

Nicolini, D. (2009) 'Articulating Practice through the Interview to the Double', Management Learning 40(2): 195-212.

Nutley, S. M., Walter, I. and Davies, H. T. O. (2007) Using Evidence: How Research Can Inform Public Services, Bristol, U.K: Policy Press.

Oliver, K. and Cairney, P. (2019) 'The dos and don'ts of influencing policy: a systematic review of advice to academics', Palgrave Communications 5(1): 1-11.

Oliver, K., Innvar, S., Lorenc, T., Woodman, J. and Thomas, J. (2014) 'A systematic review of barriers to and facilitators of the use of evidence by policymakers', BMC Health Services Research 14(1): 2.

Oreszczyn, S. (2005) 'GM crops in the United Kingdom: precaution as process', Science and Public Policy 32(4): 317-324.

Page, E. C. (2003) 'The Civil Servant as Legislator: Law Making in British Administration', Public Administration 81(4): 651-679.

Page, E. C. (2010) 'Bureaucrats and expertise: Elucidating a problematic relationship in three tableaux and six jurisdictions', Sociologie du Travail 52(2): 255-273.

Parkhurst, J. O. (2017) The politics of evidence: from evidence-based policy to the good governance of evidence, Abingdon, Oxon ; New York, NY: Routledge.

Pielke, R. A. (2007) The Honest Broker: Making Sense of Science in Policy and Politics, Cambridge University Press.

Sanderson, I. (2002) 'Evaluation, Policy Learning and Evidence-Based Policy Making', Public Administration 80(1): 1-22.

Schenkel, R. (2010) 'The Challenge of Feeding Scientific Advice into Policy-Making', Science 330(6012): 1749-1751.

Schmidt, J. A. (2014) 'Engineering as willing', in P. E. Michelfelder, D. E. Goldberg, N. McCarthy, and D. P. Michelfelder (eds). Philosophy and Engineering: Reflections on Practice, Principles and Process. Dordrecht, NETHERLANDS, THE: Springer, available at http://ebookcentral.proquest.com/lib/ucl/detail.action?doclD=1636829 (accessed January 2020).

Schwach, V. et al. (2007) 'Policy and knowledge in fisheries management: a policy brief', ICES Journal of Marine Science: Journal du Conseil 64(4): 798-803. 
Shove, E. (1998) 'Gaps, barriers and conceptual chasms: theories of technology transfer and energy in buildings', Energy Policy 26(15): 1105-1112.

Sovacool, B. K. (2014) 'Diversity: Energy studies need social science', Nature News 511(7511): 529.

Sovacool, B. K. et al. (2015) 'Integrating social science in energy research', Energy Research \& Social Science 6: 95-99.

Spruijt, P. et al. (2014) 'Roles of scientists as policy advisers on complex issues: A literature review', Environmental Science \& Policy 40: 16-25.

Stanley, M. (2000) How to be a Civil Servant, London: Politico's Publishing Ltd.

Weale, A. (2001) 'Science advice, democratic responsiveness and public policy', Science and Public Policy 28(6): 413-421.

Whitaker, E. M. and Atkinson, P. (2019) 'Authenticity and the interview: a positive response to a radical critique', Qualitative Research 1468794118816885.

Wilkinson, K. (2011) 'Organised Chaos: An Interpretive Approach to Evidence-Based Policy Making in Defra', Political Studies 59(4): 959-977.

\footnotetext{
'All Scopus searches reported used the default 'title, abstract, keyword' search, time frame and source types. All searches were carried out in early June 2019.

ii Engineering the Future - a vision for the future of UK engineering (undated, but likely 2010). Available at: https://www.engc.org.uk/EngCDocuments/Internet/Website/Engineering\%20the\%20future\%20\%20Manifesto.pdf Accessed 15 January 2020. See Section 5.

iii See: https://www.raeng.org.uk/education/what-is-engineering Accessed 5 June 2019.

iv Emphasis added. See: https://www.gov.uk/government/collections/the-analysis-function-in-government Accessed 3 Sep 2019.

' For example, the current 'About us' webpage for the UK's Department of Business, Energy and Industrial Strategy (BEIS - the Department into which DECC was absorbed in 2016) says "We are building an economy that works for everyone, so that there are great places in every part of the UK for people to work and for businesses to invest, innovate and grow." https://www.gov.uk/government/organisations/department-forbusiness-energy-and-industrial-strategy/about Accessed 3 Sep 2019

vi For more information about UK Civil Service Gradestructures, the independent UK think tank 'The Institute for Government' has a useful resource: https://www.instituteforgovernment.org.uk/explainers/gradestructures-civil-service
} 